\title{
COVID-19 severity and age increase the odds of delirium in hospitalized adults with confirmed SARS-CoV-2 infection: a cohort study
}

Sara C. LaHue ${ }^{1,2^{*}}$, Danielle P. Escueta ${ }^{3}$, Elan L. Guterman ${ }^{1,2}$, Kanan Patel ${ }^{4}$, Krista L. Harrison ${ }^{4,5}$, W. John Boscardin ${ }^{6}$, Vanja C. Douglas ${ }^{1,2}$ and John C. Newman ${ }^{4,7}$

\begin{abstract}
Background: Despite recognition of the neurologic and psychiatric complications associated with SARS-CoV-2 infection, the relationship between coronavirus disease 19 (COVID-19) severity on hospital admission and delirium in hospitalized patients is poorly understood. This study sought to measure the association between COVID-19 severity and presence of delirium in both intensive care unit (ICU) and acute care patients by leveraging an existing hospital-wide systematic delirium screening protocol. The secondary analyses included measuring the association between age and presence of delirium, as well as the association between delirium and safety attendant use, restraint use, discharge home, and length of stay.

Methods: In this single center retrospective cohort study, we obtained electronic medical record (EMR) data using the institutional Epic Clarity database to identify all adults diagnosed with COVID-19 and hospitalized for at least 48-h from February 1-July 15, 2020. COVID-19 severity was classified into four groups. These EMR data include twice-daily delirium screenings of all patients using the Nursing Delirium Screening Scale (non-ICU) or CAM-ICU (ICU) per existing hospital-wide protocols.

Results: A total of 99 patients were diagnosed with COVID-19, of whom 44 patients required ICU care and 17 met criteria for severe disease within 24-h of admission. Forty-three patients (43\%) met criteria for delirium at any point in their hospitalization. Of patients with delirium, 24 (56\%) were 65 years old or younger. After adjustment, patients meeting criteria for the two highest COVID-19 severity groups within 24-h of admission had 7.2 times the odds of having delirium compared to those in the lowest category [adjusted odds ratio (aOR) 7.2; 95\% confidence interval (Cl) 1.9, $27.4 ; P=0.003$ ]. Patients $>65$ years old had increased odds of delirium compared to those $<45$ years old (aOR 8.7; 95\% $\mathrm{Cl} 2.2,33.5 ; P=0.003)$. Delirium was associated with increased odds of safety attendant use (aOR $4.5 ; 95 \% \mathrm{Cl} 1.0,20.7$; $P=0.050)$, decreased odds of discharge home (aOR 0.2; $95 \% \mathrm{Cl} 0.06,0.6 ; P=0.005)$, and increased length of stay (aOR $7.5 ; 95 \% \mathrm{Cl} 2.0,13 ; P=0.008)$.
\end{abstract}

Conclusions: While delirium is common in hospitalized patients of all ages with COVID-19, it is especially common in those with severe disease on hospital admission and those who are older. Patients with COVID-19 and delirium,

\footnotetext{
*Correspondence: sara.lahue@ucsf.edu

${ }^{1}$ Department of Neurology, School of Medicine, University of California,

San Francisco, CA, USA

Full list of author information is available at the end of the article
} original author(s) and the source, provide a link to the Creative Commons licence, and indicate if changes were made. The images or other third party material in this article are included in the article's Creative Commons licence, unless indicated otherwise in a credit line to the material. If material is not included in the article's Creative Commons licence and your intended use is not permitted by statutory regulation or exceeds the permitted use, you will need to obtain permission directly from the copyright holder. To view a copy of this licence, visit http://creativecommons.org/licenses/by/4.0/. The Creative Commons Public Domain Dedication waiver (http://creativeco mmons.org/publicdomain/zero/1.0/) applies to the data made available in this article, unless otherwise stated in a credit line to the data. 
compared to COVID-19 without delirium, are more likely to require safety attendants during hospitalization, less likely to be discharged home, and have a longer length of stay. Individuals with COVID-19, including younger patients, represent an important population to target for delirium screening and management as delirium is associated with important differences in both clinical care and disposition.

Keywords: Delirium, Encephalopathy, COVID-19, Hospitalization, Restraints, Safety attendants, Hospital discharge, Patient outcomes

\section{Introduction}

Delirium is a life-threatening acute disturbance in mental status affecting more than 2.6 million hospitalized older adults in the United States annually [1]. Delirium is known to be more common in older adults and those requiring intensive care unit (ICU) care [2, 3]. Delirium is also associated with many poor clinical outcomes, including long-term cognitive decline and increased mortality $[4,5]$. The severity and inflammatory/vascular pathophysiology of coronavirus disease 19 (COVID-19) suggest that this virus could be particularly deliriogenic, with important implications for the long-term impact of the pandemic $[6,7]$. Indeed, delirium is a common complication in patients with COVID-19 and has been shown to be associated with an increased risk of mortality in these patients [8-10]. While the neurological and psychological complications associated with coronavirus disease 19 (COVID-19) are well recognized [11], many studies investigating the impact of COVID-19 on delirium frequency have centered on ICU populations [10,12] or do not clearly state the criteria for delirium diagnosis [9]. This study sought to identify the association between COVID-19 severity or age with the presence of delirium in hospitalized adults using a systematic in person delirium screening program. In addition, we measured the association between age and presence of delirium, as well as the association between delirium and safety attendant use, restraint use, discharge home, and length of stay in this population of patients with COVID-19.

\section{Methods}

\section{Study design}

For this retrospective cohort study, we obtained electronic medical record (EMR) data using the institutional Epic Clarity database to identify all adult patients hospitalized at the single center for at least $48 \mathrm{~h}$ and diagnosed with COVID-19 between February 1, 2020-July 15, 2020. COVID-19 diagnosis was identified using International Classification of Diseases, Tenth Revision code U07.1, which was assigned to all individuals diagnosed with COVID-19 using reverse transcriptase polymerase chain reaction for SARS-CoV-2. COVID-19 infection severity classification was based on World Health Organization criteria within $24 \mathrm{~h}$ of admission [13]. COVID-19 severity was classified as follows, from most mild (group 1) to most severe (group 4): hospitalized, no oxygen therapy; oxygen by mask or nasal prongs; non-invasive ventilation or high-flow oxygen; intubation and mechanical ventilation. This study was performed in accordance with the Declaration of Helsinki and was approved by the University of California, San Francisco Institutional Review Board (\#20-30,960).

\section{Delirium metrics}

Delirium was defined as a positive Confusion Assessment Method for ICU (CAM-ICU) [14], or a Nursing Delirium Screening Scale (NuDESC) score of 2 or higher [15], at any point during the hospitalization. The presence of delirium was identified using EMR data generated by preexisting systematic, hospital-wide screening protocols. Our study leveraged the UCSF Delirium Care Pathway [16], which is an interdisciplinary, multicomponent pathway that includes in person twice-daily bedside delirium screening, as well as standardized, evidence-based nonpharmacologic delirium management recommendations.

Under the hospital-wide Delirium Care Pathway, trained bedside nursing staff assess delirium in all hospitalized patients every 12-h shift using tools developed for clinical screening purposes. Delirium in non-ICU acute care patients is assessed using the Nursing Delirium Screening Scale (NuDESC), a validated screening tool for non-ICU hospitalized patients [15]. NuDESC is scored $0-2$ via standardized criteria in each of five categories: disorientation, inappropriate behavior, inappropriate communication, hallucinations, and psychomotor retardation. A positive screen is defined by a score of 2 or more. In ICU patients, delirium screenings use the ICUspecific Confusion Assessment Method (CAM-ICU), which assesses acute change or fluctuating course, inattention, disorganized thinking, and level of consciousness [14]. CAM-ICU is also performed in person by the bedside nurse each shift; a positive screen is determined by the bedside nurse using the validated CAM-ICU algorithm [14]. Both NuDESC and CAM-ICU results are entered into the EMR by bedside nursing staff each shift. At least $86 \%$ of patients underwent on average two in person delirium screens a day during the study period, which increased to $94 \%$ of patients when assessing 
screening compliance only during full calendar days (e.g., excluding day of admission or discharge), which is similar to prior compliance assessments [16]. The remaining patients underwent on average at least one in person delirium screen a day despite intensive hospital-wide infection control isolation protocols for patients with COVID-19. Of all patients, only two patients had a day without a delirium screen; however, both patients were diagnosed with delirium during their hospitalizations through other screens, so this did not affect our identification of delirium in these patients.

\section{Outcomes and statistical analysis}

The primary analysis was measuring the association between COVID-19 severity and presence of delirium during hospitalization. Secondary analyses included measuring the association between age and presence and delirium; the associations between delirium and safety attendant use, restraint use, discharge to home as opposed to an alternative location, and length of stay were also assessed. In addition to being a hospital-wide quality improvement metric [16], we were particularly interested in restraint use since, due to infection control measures limiting the time staff could safely spend with COVID-19 patients, restraint use may be more common in adults with delirium during the pandemic.

Differences between delirious and non-delirious patients were calculated using Wilcoxon rank-sum tests/t-tests or chi-square tests/Fisher's exact tests where appropriate. Multivariable logistic regression models assessed the association between initial COVID-19 severity and delirium, as well as the association between delirium and secondary outcomes, adjusting for clinical characteristics. Multivariable linear regression model assessed the association between length of stay and delirium, adjusting for clinical characteristics. To reduce overfitting from limited sample size, COVID-19 severity scores 3 and 4 were combined into one variable, and the variables race and ethnicity were combined into three categories (Table 1). To limit the number of variables in the model, we adjusted only for clinical characteristics that were significant in the univariate analysis: age, race, ethnicity, and COVID-19 severity classification. Due to high correlation and relationship between COVID-19 severity and ICU contact, ICU contact was not included separately in the model. The assumption of linearity for age was evaluated and confirmed. To better illustrate the distribution of delirium within different age groups, the age category was divided into three variables, with model results similar when using age as either a linear or categorized variable. Statistical analysis was performed using Stata 16.1 (StataCorp LLC, College Station, Texas) and SAS version 9.4 (SAS Institute, Inc., Cary, NC).
Table 1 Demographics and admission clinical characteristics of hospitalized patients with COVID-19

\begin{tabular}{|c|c|c|}
\hline & $\begin{array}{l}\text { No Delirium } \\
(N=56)\end{array}$ & $\begin{array}{l}\text { Delirium } \\
(N=43)\end{array}$ \\
\hline Age in Years, Median (IQR) & $47.5(37.5-63)$ & $62(47-81)$ \\
\hline \multicolumn{3}{|l|}{ Age in Years, N (\%) } \\
\hline$<45$ years & $25(45 \%)$ & $7(16 \%)$ \\
\hline $45-65$ years & $22(39 \%)$ & $17(40 \%)$ \\
\hline$>65$ years & $9(16 \%)$ & $19(44 \%)$ \\
\hline Female Sex, N (\%) & $16(29 \%)$ & $13(30 \%)$ \\
\hline \multicolumn{3}{|l|}{ Race, N (\%) } \\
\hline Asian & $8(14 \%)$ & $11(26 \%)$ \\
\hline Black & $6(11 \%)$ & $5(12 \%)$ \\
\hline Native Hawaiian or Other Pacific Islander & $1(2 \%)$ & $1(2 \%)$ \\
\hline Other & $25(45 \%)$ & $8(19 \%)$ \\
\hline Unknown & $4(7 \%)$ & $1(2 \%)$ \\
\hline White & $12(21 \%)$ & $17(40 \%)$ \\
\hline \multicolumn{3}{|l|}{ Ethnicity, N (\%) } \\
\hline Hispanic or Latino & $24(43 \%)$ & $9(21 \%)$ \\
\hline Not Hispanic or Latino & $29(52 \%)$ & $32(74 \%)$ \\
\hline Unknown & $3(5 \%)$ & $2(5 \%)$ \\
\hline \multicolumn{3}{|l|}{ Race and Ethnicity combined, N (\%) } \\
\hline Hispanic or Latino & $24(43 \%)$ & $9(21 \%)$ \\
\hline White Non-Hispanic & $10(18 \%)$ & $14(33 \%)$ \\
\hline Non-White Non-Hispanic & $22(39 \%)$ & $20(46 \%)$ \\
\hline \multicolumn{3}{|l|}{ Admission Source, N (\%) } \\
\hline Home & $40(71 \%)$ & $21(49 \%)$ \\
\hline Skilled Nursing Facility & $2(4 \%)$ & $5(12 \%)$ \\
\hline Outside Hospital Transfer & $14(25 \%)$ & $17(40 \%)$ \\
\hline \multicolumn{3}{|l|}{ Any Intensive Care Unit Contact } \\
\hline Yes & $14(25 \%)$ & $30(70 \%)$ \\
\hline No & $42(75 \%)$ & $32(30 \%)$ \\
\hline \multicolumn{3}{|c|}{ COVID-19 Severity Classification within 24-Hours of Admission" ${ }^{a}$ N (\%) } \\
\hline 1-hospitalized, no oxygen therapy & $20(36 \%)$ & $8(19 \%)$ \\
\hline 2-oxygen by mask or nasal prongs & $24(43 \%)$ & $18(42 \%)$ \\
\hline $\begin{array}{l}\text { 3-non-invasive ventilation or high-flow } \\
\text { oxygen }\end{array}$ & $7(13 \%)$ & $5(12 \%)$ \\
\hline 4-intubation and mechanical ventilation & $5(9 \%)$ & $12(28 \%)$ \\
\hline $\begin{array}{l}\text { Hospital length of stay (days), median } \\
\text { (q1-q3) }\end{array}$ & $7.5(4-13)$ & $14(11-27)$ \\
\hline
\end{tabular}

${ }^{a}$ COVID-19 severity was classified as follows: 1 - hospitalized, no oxygen therapy; 2 - oxygen by mask or nasal prongs; 3 - non-invasive ventilation or high-flow oxygen; 4 - intubation and mechanical ventilation

\section{Results}

Participant demographics and clinical characteristics

A total of 99 patients were diagnosed with COVID-19, of whom 44 patients required ICU care and 17 met criteria for severity scores 3 and 4 combined within 24-h of admission (Table 1). In total, 43 patients (43\%) were delirious during hospitalization. Thirty patients $(70 \%)$ with exposure to ICU-level care were delirious during 
their hospitalization, compared with 14 (25\%) patients with no ICU exposure $(\mathrm{p}<0.001)$. Of patients more than 65 years old, $19(68 \%)$ were delirious, compared with 24 (34\%) patients 65 years old and under $(P=0.01)$.

\section{COVID-19 severity and delirium}

Patients with the highest level of COVID-19 severity (groups 3 and 4 combined) within $24 \mathrm{~h}$ of admission had 7.2 times the odds of being delirious compared to those with the lowest level of COVID-19 severity (aOR 7.2; 95\% CI 1.9, 27.4; $P=0.003$; Table 2). Delirium was more common in older adults, where patients $>65$ years old had 8.7 times the odds of having delirium compared to those $<45$ years (aOR 8.7; 95\% CI 2.2, 33.5; $P=0.003$; Table 2).

\section{Clinical outcomes by delirium status}

The adjusted odds of safety attendant use were 4.5 times higher for those with delirium after adjusting for age, race/ethnicity and COVID-19 severity (aOR 4.5; 95\% CI 1.0, 20.7; $P=0.050$; Table 3). The odds of being discharged to a location other than home were 5 times lower for patients with delirium compared with patients without delirium (aOR 0.2; 95\% CI 0.06, 0.6; $P=0.005$; Table 3$)$. The length of stay was 7.5 days longer for those with delirium (adjusted parameter estimate 7.50; 95\% CI 2.0, 13.0; $P=0.008$; Table 3 ). Since there were only three patients without delirium with restraints, conclusions regarding the association between delirium and restraint use could not be reached with this small sample size. However, it is worth noting that only $5 \%$ of patients without delirium were restrained during hospitalization compared with $58 \%$ of patients with delirium.

\section{Discussion}

In this study of 99 adults hospitalized with COVID19 at an academic medical center in Northern California, we found that admission COVID-19 severity

Table 2 Odds of delirium based on COVID-19 severity and age among patients with COVID-19

\begin{tabular}{|c|c|c|}
\hline Characteristic & $\begin{array}{l}\text { Unadjusted Odds ratio }(95 \% \mathrm{Cl}=95 \% \text { confidence } \\
\text { interval); } P \text {-value }\end{array}$ & $\begin{array}{l}\text { Adjusted Odds ratio }(95 \% \\
\mathrm{Cl}=95 \% \text { confidence interval); } \\
P \text {-value }\end{array}$ \\
\hline \multicolumn{3}{|l|}{ COVID-19 severity* } \\
\hline 1-hospitalized, no oxygen therapy & ref & ref \\
\hline $\begin{array}{l}\text { 2-oxygen by mask or nasal prongs vs. 1-hospitalized, } \\
\text { no oxygen therapy }\end{array}$ & $1.9(0.7,5.2) ; p=1.0$ & $2.0(0.6,6.5) ; p=0.6^{*}$ \\
\hline $\begin{array}{l}\text { 3/4- ventilation or high-flow oxygen vs. 1-hospital- } \\
\text { ized, no oxygen therapy }\end{array}$ & $3.5(1.2,10.7) ; p=0.04$ & $7.2(1.9,27.4) ; p=0.003^{*}$ \\
\hline \multicolumn{3}{|l|}{$\mathrm{Age}^{* *}$} \\
\hline Age $<45$ years & ref & ref \\
\hline Age $45-65$ years & $\begin{array}{l}2.8(0.97,7.9) \\
P=0.99\end{array}$ & $\begin{array}{l}2.4(0.7,7.5) \\
P=0.64^{* *}\end{array}$ \\
\hline Age $>65$ years & $\begin{array}{l}7.5(2.4,23.9) \\
P=0.002\end{array}$ & $\begin{array}{l}8.7(2.2,33.5) \\
P=0.003^{* *}\end{array}$ \\
\hline
\end{tabular}

* Adjusted for age, race, ethnicity

${ }^{* *}$ Adjusted for age, race, ethnicity, and COVID-19 severity classification

Table 3 Outcomes associated with delirium among patients with COVID-19

\begin{tabular}{|c|c|c|c|c|}
\hline Outcome & $\begin{array}{l}\text { No Delirium }(N=56) \text {, } \\
\text { n (\%) or median days } \\
(q 1-q 3)\end{array}$ & $\begin{array}{l}\text { Delirium }(N=43), n(\%) \\
\text { or median days (q1-q3) }\end{array}$ & $\begin{array}{l}\text { Unadjusted Model Result } \\
\text { (95\% confidence interval); } \\
P \text {-value }\end{array}$ & $\begin{array}{l}\text { Adjusted* Model Result } \\
\text { ( } 95 \% \text { confidence interval); } \\
P \text {-value }\end{array}$ \\
\hline $\begin{array}{l}\text { Safety attendant use odds } \\
\text { ratio^} \wedge\end{array}$ & $4(7 \%)$ & $12(28 \%)$ & $5.0(1.5,17.0) ; P=0.009$ & $4.5(1.0,20.7) ; P=0.050$ \\
\hline $\begin{array}{l}\text { Discharge to home odds } \\
\text { ratio }\end{array}$ & $47(84 \%)$ & $17(40 \%)$ & $0.1(0.05,0.3) ; P<0.0001$ & $0.2(0.06,0.6) ; P=0.005$ \\
\hline $\begin{array}{l}\text { Length of stay, median days } \\
\text { (q1-q3) parameter estimate }\end{array}$ & $7.5(4-13)$ & $14(11-27)$ & $10.3(5.3,15.3) ; P<0.0001$ & $7.5(2.0,13.0) ; P=0.008$ \\
\hline
\end{tabular}

\footnotetext{
* Adjusted for age, race, ethnicity, and COVID-19 severity classification

${ }^{\wedge}$ Safety attendant use and Discharge to home $=$ Odds ratio ( $95 \%$ confidence interval); $p$-value

\# Length of stay = parameter estimate ( $95 \%$ Confidence interval); $p$-value
} 
was independently associated with increased odds of delirium during hospitalization. This cohort included patients cared for on the acute care medical ward as well as those in the ICU for any portion of their stay, which builds on previously published studies investigating delirium in ICU $[10,12,17]$ or emergency department [18] populations in isolation. Importantly, our data suggest that delirium may be underdiagnosed in patients with COVID-19 as our observed delirium frequency was comparable or higher than reported in ICU studies [12, 17] or in older adults upon ED presentation [18]. These data emphasize the urgent need to expand the designation of patient populations at risk for delirium during the COVID-19 pandemic.

We found a high frequency of delirium even in this relatively young cohort, which counters established demographic norms for delirium. While patients with severe COVID-19 were most likely to develop delirium, we found that the frequency of delirium was high even in younger patients. Currently, many hospital-based programs that assess delirium risk and screen for active delirium are targeted to older adults who may benefit the most. Further, studies investigating delirium epidemiology and patient outcomes are often limited to older adult populations; indeed, our recent study investigating clinical outcomes following initiation of our hospitalwide delirium care pathway only included patients who were at least 50 years old [11]. However, here we demonstrated that delirium was also a common complication for younger adults. This suggests that the scales of delirium risk in adults with COVID-19 tip away from age and instead weigh more heavily on the impact of COVID-19 pathophysiology, such as its unique neuroinflammatory cascade serving as a delirium generator. These results suggest that our paradigms of delirium risk in hospitalized adults with COVID-19 should be more inclusive and incorporate rigorous screening for people of all ages.

The primary strength of this study is its ability to leverage the comprehensive, hospital-wide delirium screening program and data collection tools that predated the COVID-19 pandemic. All delirium screens were in person. EMR delirium screening data included all COVID-19 inpatients throughout their stay, including both ICU and non-ICU periods, to comprehensively define delirium rates for the complete hospitalization. We identified delirium cases during a period when COVID-19 hospitalizations were common but did not overwhelm standard screening protocols, though intensive hospital-wide infection control isolation protocols were in effect for patients with COVID-19. Despite these protocols, at least $86 \%$ of our study population underwent on average two in person delirium screens a day during the study period, which increased to $94 \%$ of patients when assessing screening compliance only during full calendar days. Of all patients, only two patients had a day without a delirium screen; however, both patients were diagnosed with delirium during their hospitalizations using the other screens, so this did not affect our identification of delirium in these patients.

Our EMR-based retrospective study has several limitations. It is possible that the NuDESC screening tool for non-ICU patients underestimated delirium cases, as NuDESC has a high specificity but lower sensitivity compared with more intensive delirium diagnostic methods. Further, we cannot exclude the possibility that part of the association between COVID-19 severity and delirium was due to the use of different screening tools in the ICU (e.g., CAM-ICU), where COVID-19 is more severe, compared with the acute care units (e.g., NuDESC), where COVID-19 is less severe, if the CAMICU is more sensitive than the NuDESC. This potential underestimate may be somewhat compensated for by the regularity and frequency of NuDESC screening and would only mean that our already-high measurements of delirium in COVID-19 may be even higher. However, the NuDESC is a well-validated time-efficient delirium screening tool for nursing staff and so was ideal during a time when increased patient load risked compromising established delirium screening protocols. Further, the higher rate of delirium in these patients could be due to factors other than COVID-19. With longer lengths of stay, the patient becomes at risk for delirium due to causes other than the reason for the initial hospitalization, which in our cohort was COVID-19, and because more severe COVID-19 is likely to lead to longer LOS, the higher rate of delirium in these patients could be due to factors other than COVID-19. However, the specificity of the delirium screens used in this study is high $[14,19]$, so false positives are unlikely. In addition, presence of delirium has been shown to be associated with longer length of stay, and interventions that target delirium reduce length of stay [16]. Because we did not measure the date on which delirium was first diagnosed in each of our patients, we are unable to distinguish between delirium that started on hospital day 2 (and thus was more likely due to COVID-19) and delirium that started on hospital day 30 (and thus was more likely due to a hospital acquired complication). This temporal trend will be an important factor to include in future studies. Additionally, our sample size is relatively small due to relatively low local hospitalization rates at this early phase of the pandemic, though the sample size is similar to previously published studies and sufficiently powered to detect relevant associations. 


\section{Conclusions}

Delirium is common in hospitalized patients with COVID-19, especially in those with severe disease on hospital admission, and even in younger patients. Delirium in patients with COVID-19 was associated with several negative outcomes. Individuals with COVID-19, including younger patients, represent an important population to target for delirium screening and management as delirium is associated with important differences in both clinical care and disposition.

\section{Abbreviations}

aOR: Adjusted Odds Ratio; Cl: Confidence Interval; CAM-ICU: Confusion Assessment Method ICU; COVID-19: Coronavirus disease 19; EMR: Electronic medical record; ICU: Intensive Care Unit; NuDESC: Nursing Delirium Screening Scale; OR: Odds Ratio.

\section{Acknowledgements}

We would like to acknowledge Joy Youn for her assistance with data collection

\section{Authors' contributions}

SCL made substantial contributions to the conception and design of the work: acquisition, analysis and interpretation of data; and drafted the work. DPE made substantial contributions to the conception and design of the work; acquisition, analysis and interpretation of data; and substantially revised the work. EG made substantial contributions to the design of the work; analysis and interpretation of data; and substantially revised the work. KP made substantial contributions to the design of the work; acquisition, analysis and interpretation of data; and substantially revised the work. KLH made substantial contributions to the design of the work; analysis and interpretation of data; and substantially revised the work. WJB made substantial contributions to the analysis and interpretation of data; and substantially revised the work. VCD made substantial contributions to the design of the work; analysis and interpretation of data; and substantially revised the work. JCN made substantial contributions to the conception and design of the work; interpretation of data; and substantially revised the work. All authors have approved the submitted version and have agreed to both to be personally accountable for the author's own contributions and to ensure that questions related to the accuracy or integrity of any part of the work, even ones in which the author was not personally involved, are appropriately investigated, resolved, and the resolution documented in the literature.

\section{Funding}

$\mathrm{SCL}$ receives funding from the National Institute on Aging (R03AG074035), Larry L. Hillblom Foundation (A137420), and the Bakar Aging Research Institute. ELG receives funding from the National Institute of Neurological Disorders and Stroke (1K23NS116128-01) and the National Institute on Aging (5R01AG056715), American Academy of Neurology as well as consulting fees from Marinus Pharmaceuticals, Inc. VCD is supported by the Sara \& Evan Williams Foundation Endowed Neurohospitalist Chair. SCL, KLH, KP, WJB, and JCN are supported by the UCSF Claude D. Pepper Older Americans Independence Center funded by National Institute on Aging (P30 AG044281). KLH is also supported by funding from a National Institute on Aging Mentored Research Scientist Development Award (K01 AG059831). JCN is also supported by National Institute on Aging R01 AG068025.

\section{Availability of data and materials}

The datasets generated and analyzed during the current study are not publicly available due to them containing information that could compromise participant privacy. The data however are available from the corresponding author $(\mathrm{SCL})$ on reasonable request.

\section{Declarations}

\section{Ethics approval and consent to participate}

This study was performed in accordance with the Declaration of Helsinki and was approved by the University of California, San Francisco (UCSF) Institutional Review Board (\#20-30960). As this study was deemed minimal risk by the UCSF Institutional Review Board, the requirement for informed consent to participate was waived.

\section{Consent for publication}

Not applicable.

\section{Competing interests}

The authors have no competing interests to disclose.

\section{Author details}

${ }^{1}$ Department of Neurology, School of Medicine, University of California, San Francisco, CA, USA. ${ }^{2}$ Department of Neurology, Weill Institute for Neurosciences, University of California, San Francisco, CA, USA. ${ }^{3}$ School of Medicine, University of California, San Francisco, CA, USA. ${ }^{4}$ Division of Geriatrics, Department of Medicine, School of Medicine, University of California, San Francisco, CA, USA. ${ }^{5}$ Philip R. Lee Institute for Health Policy Studies, University of California, San Francisco, CA, USA. ${ }^{6}$ Department of Epidemiology \& Biostatistics, School of Medicine, University of California, San Francisco, CA, USA. ${ }^{7}$ Buck Institute for Research On Aging, Novato, CA, USA.

Received: 2 March 2021 Accepted: 23 February 2022

Published online: 28 February 2022

\section{References}

1. Oh ES, Fong TG, Hshieh TT, Inouye SK. Delirium in Older Persons: Advances in Diagnosis and Treatment. JAMA. 2017;318(12):1161-74.

2. Marcantonio ER. Delirium in Hospitalized Older Adults. N Engl J Med. 2017;377(15):1456-66.

3. Salluh JI, Soares M, Teles JM, Ceraso D, Raimondi N, Nava VS, Blasquez P, Ugarte S, Ibanez-Guzman C, Centeno JV, et al. Delirium epidemiology in critical care (DECCA): an international study. Crit Care. 2010;14(6):R210.

4. Ely EW, Shintani A, Truman B, Speroff T, Gordon SM, Harrell FE Jr, Inouye SK, Bernard GR, Dittus RS. Delirium as a predictor of mortality in mechanically ventilated patients in the intensive care unit. JAMA. 2004;291(14):1753-62.

5. Siddiqi N, House AO, Holmes JD. Occurrence and outcome of delirium in medical in-patients: a systematic literature review. Age Ageing. 2006:35(4):350-64.

6. Wilson JE, Mart MF, Cunningham C, Shehabi Y, Girard TD, MacLullich AMJ, Slooter AJC, Ely EW. Delirium Nat Rev Dis Primers. 2020;6(1):90.

7. LaHue SC, James TC, Newman JC, Esmaili AM, Ormseth CH, Ely EW. Collaborative Delirium Prevention in the Age of COVID-19. J Am Geriatr Soc. 2020;68(5):947-9.

8. Shao SC, Lai CC, Chen YH, Chen YC, Hung MJ, Liao SC. Prevalence, incidence and mortality of delirium in patients with COVID-19: a systematic review and meta-analysis. Age Ageing. 2021;50(5):1445-53.

9. Hariyanto TI, Putri C, Hananto JE, Arisa J, Fransisca VSR, Kurniawan A. Delirium is a good predictor for poor outcomes from coronavirus disease 2019 (COVID-19) pneumonia: A systematic review, meta-analysis, and meta-regression. J Psychiatr Res. 2021;142:361-8.

10. Pun BT, Badenes R, Heras La Calle G, Orun OM, Chen W, Raman R, Simpson BK, Wilson-Linville S, HinojalOImedillo B, Vallejo de la Cueva A, et al. Prevalence and risk factors for delirium in critically ill patients with COVID-19 (COVID-D): a multicentre cohort study. Lancet Respir Med. 2021;9(3):239-50.

11. Helms J, Kremer S, Merdji H, Clere-Jehl R, Schenck M, Kummerlen C, Collange O, Boulay C, Fafi-Kremer S, Ohana M, et al. Neurologic Features in Severe SARS-CoV-2 Infection. N Engl J Med. 2020;382(23):2268-70.

12. Khan SH, Lindroth H, Perkins AJ, Jamil Y, Wang S, Roberts S, Farber M, Rahman O, Gao S, Marcantonio ER, et al. Delirium Incidence, Duration, and Severity in Critically III Patients With Coronavirus Disease 2019. Crit Care Explor. 2020;2(12):e0290. 
13. Beigel JH, Tomashek KM, Dodd LE, Mehta AK, Zingman BS, Kalil AC, Hohmann E, Chu HY, Luetkemeyer A, Kline S, et al. Remdesivir for the Treatment of Covid-19 - Final Report. N Engl J Med. 2020;383(19):1813-26.

14. Ely EW, Inouye SK, Bernard GR, Gordon S, Francis J, May L, Truman B, Speroff T, Gautam S, Margolin R, et al. Delirium in mechanically ventilated patients: validity and reliability of the confusion assessment method for the intensive care unit (CAM-ICU). JAMA. 2001;286(21):2703-10.

15. Gaudreau JD, Gagnon P, Harel F, Tremblay A, Roy MA. Fast, systematic, and continuous delirium assessment in hospitalized patients: the nursing delirium screening scale. J Pain Symptom Manage. 2005;29(4):368-75.

16. LaHue SC, Maselli J, Rogers S. Outcomes Following Implementation of a Hospital-Wide Multicomponent Delirium Care Pathway. J Hosp Med. 2021;16(7):397-403.

17. Helms J, Kremer S, Merdji H, Schenck M, Severac F, Clere-Jehl R, Studer A Radosavljevic M, Kummerlen C, Monnier A, et al. Delirium and encephalopathy in severe COVID-19: a cohort analysis of ICU patients. Crit Care. 2020;24(1):491.

18. Kennedy M, Helfand BKI, Gou RY, Gartaganis SL, Webb M, Moccia JM, Bruursema SN, Dokic B, McCulloch B, Ring H, et al. Delirium in Older Patients With COVID-19 Presenting to the Emergency Department. JAMA Netw Open. 2020;3(11):e2029540.

19. Hargrave A, Bastiaens J, Bourgeois JA, Neuhaus J, Josephson SA, Chinn J, Lee M, Leung J, Douglas V. Validation of a Nurse-Based Delirium-Screening Tool for Hospitalized Patients. Psychosomatics. 2017;58(6):594-603.

\section{Publisher's Note}

Springer Nature remains neutral with regard to jurisdictional claims in published maps and institutional affiliations.

- fast, convenient online submission

- thorough peer review by experienced researchers in your field

- rapid publication on acceptance

- support for research data, including large and complex data types

- gold Open Access which fosters wider collaboration and increased citations

- maximum visibility for your research: over $100 \mathrm{M}$ website views per year

At BMC, research is always in progress.

Learn more biomedcentral.com/submissions 